\title{
Design and Equivalent Mechanical Modeling of New Deployable Cabin Segment
}

\author{
Chuang Shi $\mathbb{D}^{1,2}$ Lingbin Zeng, ${ }^{1}$ Zijie Chen, ${ }^{2}$ Hongwei Guo $\mathbb{D}^{2}{ }^{2}$ \\ Yongyong Li, ${ }^{2}$ and Rongqiang Liu $^{2}$ \\ ${ }^{1}$ Space Structure and Mechanism Technology Laboratory of China Aerospace Science and Technology Group Co. Ltd., \\ Shanghai 201109, China \\ ${ }^{2}$ State Key Laboratory of Robotics, Harbin Institute of Technology, Harbin, China
}

Correspondence should be addressed to Hongwei Guo; guohw@hit.edu.cn

Received 10 November 2020; Revised 22 January 2021; Accepted 29 January 2021; Published 22 February 2021

Academic Editor: Gordon Huang

Copyright (c) 2021 Chuang Shi et al. This is an open access article distributed under the Creative Commons Attribution License, which permits unrestricted use, distribution, and reproduction in any medium, provided the original work is properly cited.

With the in-depth study of the construction of space bases, the demand for super-large deployable manned cabins in space is becoming increasingly urgent. In this study, a brand-new large space modular expandable cabin mechanism is proposed, and equivalent mechanical modeling research is conducted based on its modular characteristics. First, the overall configuration and the deployment driving scheme of the cabin section with the best comprehensive performance are optimized. In addition, a detailed structural design of the cabin section mechanism is carried out. Second, the mechanical equivalent continuous model of the cabin mechanism is established. The static and dynamic characteristics of the cabin mechanism are studied based on finite element simulation and equivalent model calculation, respectively. The correctness of the equivalent mechanical model established in this study is verified through comparative analysis. Finally, a scaled prototype is developed to verify the feasibility of the configuration and the new drive proposed in this study.

\section{Introduction}

With the progress of science and technology, deep-space exploration has become possible. In recent years, deep-space exploration activities have further understood the universe, the solar system, the origin and evolution of the Earth, and human life. Acquiring a more scientific understanding of outer space is of great significance. Moon landing activities and Mars exploration are the development trend of world space activities [1-4].

In the development process of the spacecraft, the rigid cabin started earlier. The main material of the rigid cabin is metal. According to different use requirements, a base can be divided into multiple cabins, and the cabins are connected [5]. The Columbus capsule has a mass of 12.8 tons and an envelope diameter of $4.5 \mathrm{~m} \mathrm{[5].} \mathrm{The} \mathrm{structural} \mathrm{design} \mathrm{of} \mathrm{the}$ Columbus capsule is divided into two layers, namely, the upper and lower layers, which are arranged with the work and the life cabin, and the circular channel is designed between the two layers [6, 7]. In December 2015, Austria, France, and Belgium jointly developed a self-expanding human survival cabin structure (SHEE) in an extreme natural environment [8]. The expanded SHEE has a maximum diameter of $6 \mathrm{~m}$ and a height of $2.8 \mathrm{~m}$, which can support the life and work of two staff members [9]. After extensive verification and testing, SHEE can be used in disaster relief, polar exploration, and manned bases on the Moon and Mars [10-12].

Compared with the traditional rigid base, the flexible deployable cabin structure made of a pressurized thin shell and membrane structure shows great advantages. In the transportation process, the folding-expansion ratio of the flexible deployable structure is higher than that of the rigid cabin. Moreover, the volume of the flexible cabin after the expansion increases functionally, and its expansion ability is far from being comparable to that of the traditional cabin structure [13]. In recent years, the inflatable cabin has been continuously developed. The concept of the inflatable cabin 
has been introduced into the fields of the Moon base, large space capsule, airlock cabin, and others [14-16]. A typical achievement is that the ILC Dover company in the United States successfully developed a vertical inflatable cabin with a double-cabin structure in 2007 [17]. In April 2016, the "Bigelow space company" successfully developed the inflatable space residence cabin (BEAM). After the fully unfolded cabin, the maximum diameter can reach $3.23 \mathrm{~m}$, and the maximum length can reach $4 \mathrm{~m}$. The volume has expanded 3.6 times from $4.5 \mathrm{~m}$ in the folded state, and the mass of the entire cabin has reached 1.4 tons, which is far less than that of the rigid cabin of the same size [18].

The development of the constructed base is another significant achievement of the manned spaceflight industry. The earliest designed cabin structure is made using threedimensional (3D) printing technology, which can be built not only on the ground but also underground or in karst caves. The material used is lunar soil. The biggest feature of the construction base is to solidify the temporary or inflatable structure using $3 \mathrm{D}$ printing technology to form a permanent structure $[19,20]$.

At present, the main form of manned spacecraft operating in the orbit is the rigid metal cabin. Although the technology is relatively mature, the large rigid sealed cabin has a large volume, difficult assembly in the orbit, heavy quality, and limited rocket envelope. Nevertheless, meeting the needs of future deep-space exploration development in terms of internal capacity and lightweight is difficult for the current cabin segment. The inflatable capsule has the characteristics of high folding ratio and lightweight, but its stiffness after deployment is relatively weak. The deployment requires an additional air source to provide the deployment power, and the gas source generation structure and curing device are complex. Furthermore, the quality is large, and meeting the use requirements of the future super-large space cabin is difficult. In this study, an expandable truss cabin is designed, which has the characteristics of lightweight, large folding ratio, reliable deployment, and large rigidity.

As a kind of deployable mechanism, the truss mechanism has abundant folding schemes and folding configurations. Chen et al. [21] obtained a flat foldable configuration with four turning points by graph theory. The rigidity of the truss mechanism can be improved by adding a prestressed cable-rod mechanism. Chen et al. [22] used the particle swarm optimization method to optimize the shape of the cable and rod. They proved that the stiffness of the truss mechanism can be optimized by adding the cable and rod reasonably. In this study, a deployable truss cable-rod cabin section is designed, which has the characteristics of lightweight, large fold-to-length ratio, reliable deployment, and high rigidity. Several jobs have been conducted about the sensors [23-25].

This study proposes a brand-new mechanism scheme of a large-scale modular deployable cabin in space. On this basis, studies on relevant aspects, such as structural design, the establishment of the equivalent mechanical model, and development and analysis of scaled prototype, are carried out. The chapter arrangement of the paper is as follows: Section 2 selects the overall configuration scheme of the cabin with the best comprehensive performance and designs the cabin structure in detail. Section 3 establishes the mechanical equivalent continuous model of the cabin mechanism. Through finite element comparative analysis, the verification of the equivalent model of the cabin system has been completed, and the reduction of the mechanical model is realized. Then, Section 4 develops the scale prototype of the deployable cabin system and the stretching mechanism prototype. The rationality of the mechanism configuration and the feasibility of the new driving mode are verified through the unfolding function experiment.

\section{Mechanism Design of the Expandable Cabin}

2.1. Optimization of Deployment Unit Scheme for Expandable Cabin. Referring to the configuration of the basic unit of the common articulated truss folding mechanism, this study selects the basic unfolding unit structure, that is, quadrangular prism. To increase the stability of the quadrangular prism truss, diagonal ropes or telescopic rod members are added to the basic deployment unit, as shown in Figure 1, which is the configuration diagram of six basic units. The solid line represents the bar, the dot represents the rotating pair, and the dotted line represents the diagonal rope. Configuration 1 is the basic deployment unit configuration of the quadrangular prism truss, and configuration 2 pulls two oblique cables on each of the four sides of the basic deployment unit. Moreover, configuration 3 pulls four oblique cables on the diagonal of the basic deployment unit, and configuration 4 adds a coplanar parallel telescopic inclined bar at the same position of each deployment unit. Then, configuration 5 adds a coplanar intersecting telescopic inclined bar to the basic deployment unit, and configuration 6 adds a heterogeneous intersecting inclined bar to the basic unit.

In Abaqus software, the finite element models of six basic deployment unit configurations are established, and the natural frequencies of the six schemes are simulated and compared. Table 1 shows the list of the analysis results.

Through the above analysis, configuration 2 is the optimal configuration and is selected as the basic component unit of the subsequent cabin mechanism.

2.2. System Scheme of the Deployable Cabin. Referring to the existing spacecraft structure and shape, the configuration principle of the cabin system is designed. The external structure used in the cabin is usually prismatic and cylindrical. The four overall schemes of quadrangular, hexagonal, octagonal, and ten prisms are compared and analyzed. Using the Abaqus software, models are established for the four configurations, and the parameters, such as outer envelope diameter, height, and material of each configuration, are completely set up for finite element analysis. To evaluate the characteristics of the four overall schemes, the evaluation index is defined: specific frequency $=$ frequency/quality. If the frequency is high, then the scheme is better. Figure 2 shows the specific frequency results of the four overall schemes. 


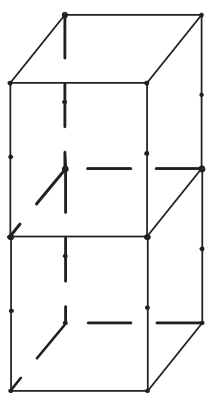

(a)

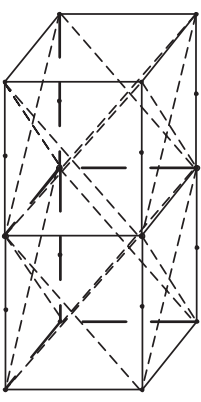

(b)

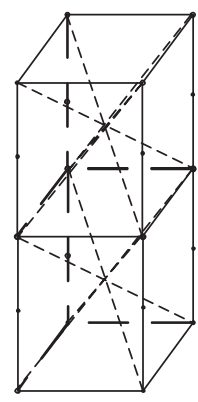

(c)

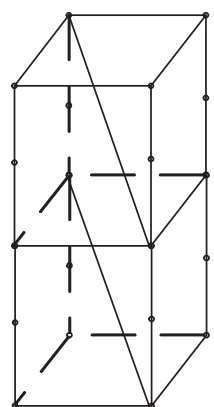

(d)

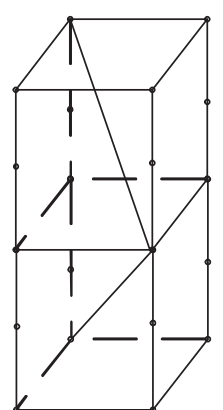

(e)

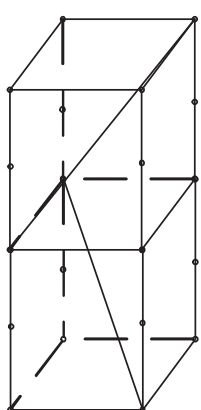

(f)

FIGURE 1: Six basic unit configurations: (a) basic unit, (b) diagonal plus oblique cable, (c) side plus oblique cable, (d) coplanar parallel slant, (e) coplanar intersecting slant, and (f) hetero planar intersecting slant.

TABLE 1: Natural frequency analysis results of six configurations.

\begin{tabular}{lccc}
\hline Unit configuration & Order $1(\mathrm{~Hz})$ & Second order $(\mathrm{Hz})$ & Order $3(\mathrm{~Hz})$ \\
\hline Configuration one & 18.010 & 18.002 & 24.135 \\
Configuration two & 35.935 & 35.936 & 48.173 \\
Configuration three & 23.454 & 29.246 & 29.247 \\
Configuration four & 16.004 & 24.184 & 28.096 \\
Configuration five & 16.776 & 24.080 & 52.235 \\
Configuration six & 21.501 & 24.055 & 29.886 \\
\hline
\end{tabular}

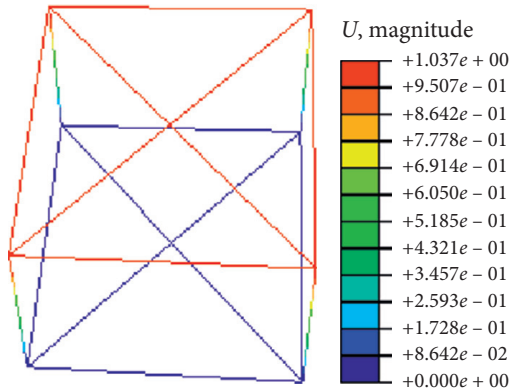

(a)

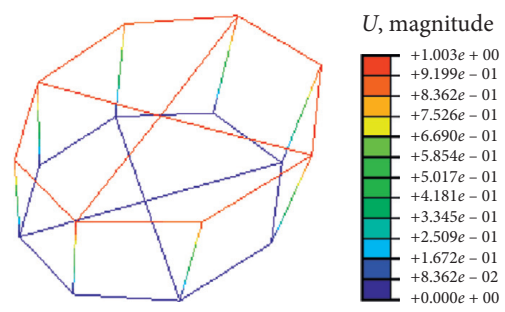

(c)

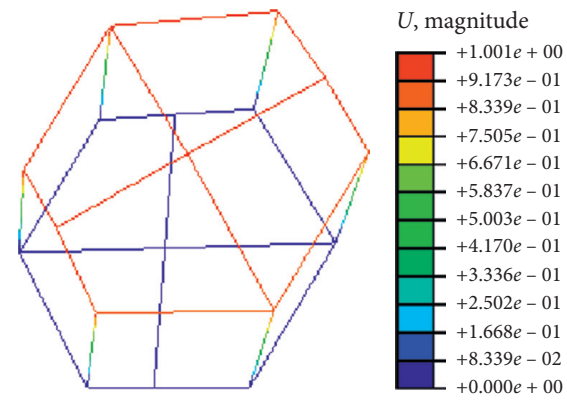

(b)

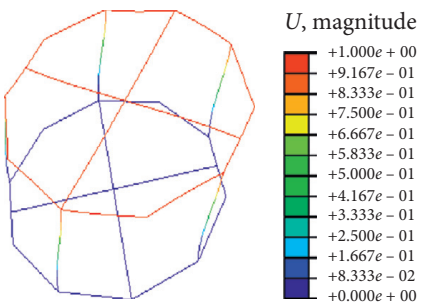

(d)

Figure 2: List of configurations: (a) quadrangular prism, (b) hexagonal prism, (c) octagonal prism, and (d) ten prism.

Figure 2 shows the configurations.

Table 2 shows that the octagonal scheme has a larger specific frequency. The overall configuration of the cabin system adopts the octagonal structure. In addition, the eight edges and the upper and lower bottom surfaces adopt edge support trusses composed of deployment units, which are connected by propulsion units at the vertices. The strengthening truss on the upper and lower bottom of the octagonal prism can be installed in two ways as shown in Figure 3. The first one is that the cross supporting trusses on the upper and lower bottom are arranged in parallel. Then, the second installation method is the cross installation of the cross support truss on the upper and lower bottom surfaces. According to the finite element simulation analysis, the fundamental frequency of the second method is relatively large. The deployable cabin system designed in this study 
TABLE 2: First third-order specific frequency of the overall configuration scheme.

\begin{tabular}{lcccc}
\hline Overall configuration & Mass $(\mathrm{kg})$ & First-order ratio frequency & Second-order ratio frequency & Third-order specific frequency \\
\hline Quadrangular prism & $1,378.67$ & 0.0033 & 0.0033 & 0.0038 \\
Hexagonal prism & $1,528.26$ & 0.0034 & 0.0035 & 0.0042 \\
Octagonal prism & $1,651.36$ & 0.0036 & 0.0036 & 0.0043 \\
Ten prism & $1,766.57$ & 0.0030 & 0.0030 & 0.0039 \\
\hline
\end{tabular}

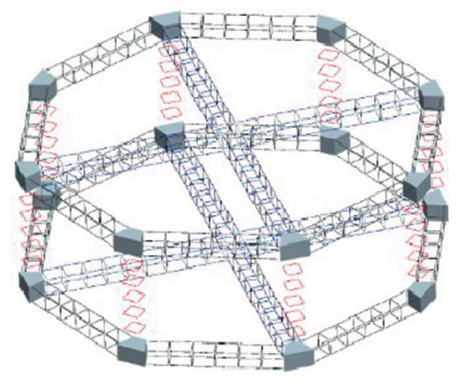

(a)

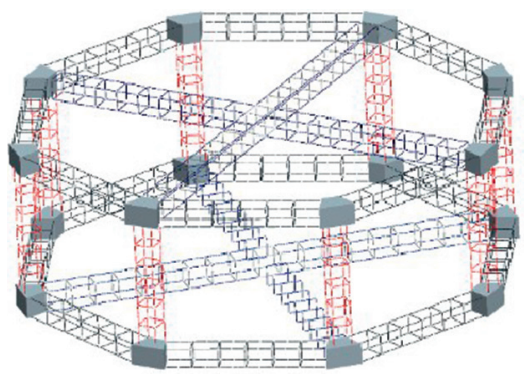

(b)

FIgURE 3: Installation scheme of cross support truss for the cabin system.

adopts the cross installation method of cross support truss on the upper and lower sides.

Figure 4 shows the sketch map of the folded and unfolded state of the entire structure of the expandable cabin.

At present, the deployable mechanism generally adopts motor, spring, and elastic rod drive, generally driven at the end of the mechanism. The driving device is complex and of high quality. This study proposes a scheme of using a microrocket engine to drive at the front end of the deployable mechanism and unfolding a large mechanism. The advantage of this engine is that it can simplify the driving scheme, and the cost is low.

As shown in Figure 5(a), the microrocket engine is arranged at the top of the cross-shaped truss in the middle. Then, the deployment is realized by dragging the crossshaped supporting truss on the upper and lower bottom surfaces. The unfolding process drives the supporting trusses of the eight edges to unfold along with the movement. Finally, the units are automatically locked after being unfolded in place.

As shown in Figure 5(b), the microrocket engine is arranged on the eight axial support trusses of octagonal prism to apply driving. To reduce the number of driving and simplify the structure, driving is applied to every other side of the octagonal prism, driving the axial unfolding mechanism to unfold sequentially and complete the locking.

\subsection{Structural Design of the Expandable Cabin}

2.3.1. Hinge Design. The limitation of the structure size after the deployment unit is folded, requiring a simple hinge structure design and high rigidity, and the self-locking function can be realized. A simple and reliable connecting hinge needs to be selected, and the rotation angle of the two members during folding is 180 . In the process of dragging and unfolding the common single-axis offset hinge, the hinge cannot pass the "dead center," due to the structural limitation, and the hinge cannot achieve a motion angle of 180 degrees; thus, the self-locking action cannot be completed. The biaxial hinge can be dragged and unfolded, but its synchronous mechanism adopts the mode of local gear meshing, which is easy to get stuck during the unfolding process.

Combined with the characteristics of the above two kinds of hinges, designing a hinge that can complete the locking in place by rotating 180 degrees along with the pulling force in the direction of the rod and does not need a synchronization mechanism is necessary. As shown in Figure 6, this study designs a double-coupled crank slider drives hinge. The structure mainly consists of the right hinge, left hinge, driving rod, dual rocker, deep groove ball bearing, locking tongue, locking hook, and others. This hinge adopts the principle of a double-coupled crank-slider mechanism to realize the expansion of the lead screw, in which the right and left hinges are used as sliders. The left hinge is installed at the bottom of the lead screw as a fixed piece, whereas the right hinge is connected to the top screw as a moving piece. Then, the driving spring is connected to the connecting rod through the driving rod to drive.

Figure 7(a) shows the hinge's unfolding process. When the hinge needs to be unfolded, the entire system is released by pressing the release mechanism, and the compression spring installed on the hinge mother hinge releases the elastic potential energy. Then, the entire hinge starts to unfold under the action of two spring elastic potential energy, and the two connecting rods move and unfold under the chute in the hinge by connecting the pin shaft. As shown in Figure 7(b), after the expansion is complete, the hinge is positioned by the conical positioning table of the right hinge and the positioning groove, and the elastic locking hook locks in the locking tongue. Considering the cumulative processing and assembly tolerance, the locking hook and the locking tongue have a 10-degree matching angle to ensure 


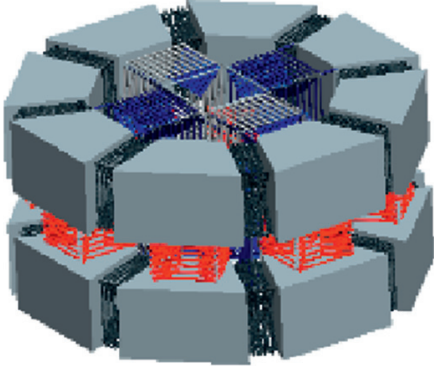

(a)

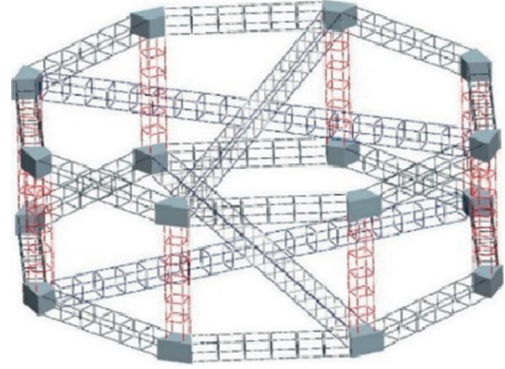

(b)

Figure 4: Schematic diagram of the overall structure of the cabin system: (a) cabin system collapse status; (b) cabin system deployment status.

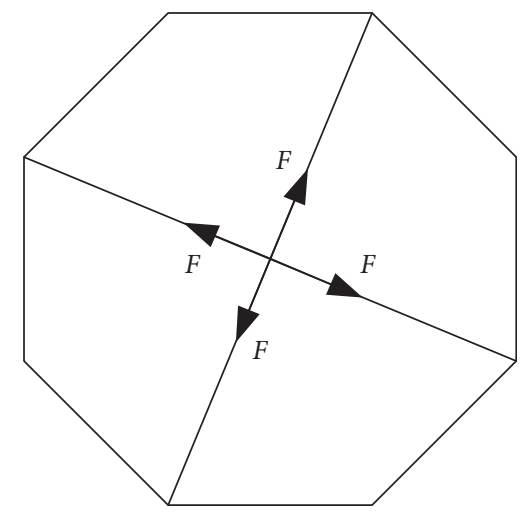

(a)

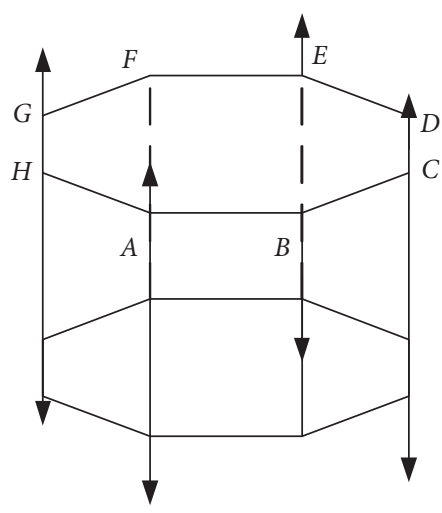

(b)

FIgURe 5: Driving principle: (a) radial drive principle; (b) axial drive principle.

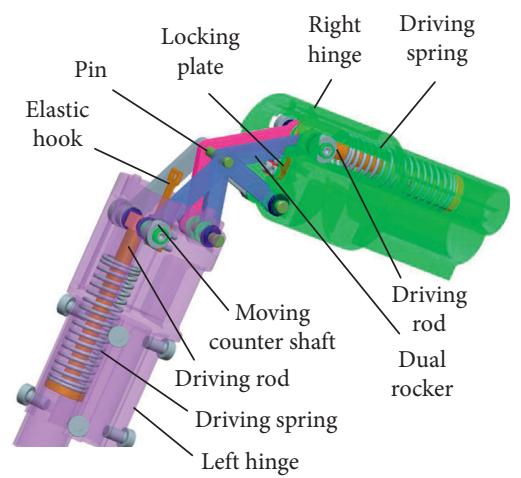

FiguRE 6: Structural diagram of expandable moment hinge.

the reliability of locking in place. In addition, an error under certain mechanical environment conditions is found.

2.3.2. Cabin Structure Design. As shown in Figure 8, the deployment unit of the expandable cabin section is mainly composed of joint hinges, diagonal ropes, carbon fiber rods, and other auxiliary components. The supporting vertical rods of the basic deployment unit are composed of joint hinges, two carbon fiber rods, and shafting rotary pair. The supporting vertical rod adopts a lateral offset folding mode to achieve a larger folding ratio and avoid interference between components. In addition, to prevent the winding phenomenon between the diagonal rope and the rod under the state of the cabin system from being folded, two rubber cable sleeves are arranged on the surface of each diagonal rope. The outer envelope diameter of the basic unit is $0.9 \mathrm{~m}$, the height of the collapsed state is $0.089 \mathrm{~m}$, and the height of the fully expanded state is $1.62 \mathrm{~m}$.

As shown in Figure 9, the envelope diameter in the folded state of the deployable cabin system is $4.9 \mathrm{~m}$ and the 


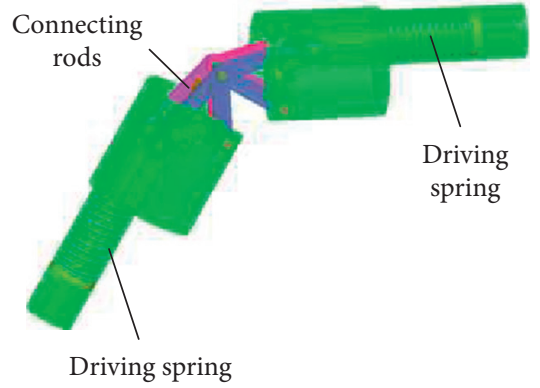

(a)

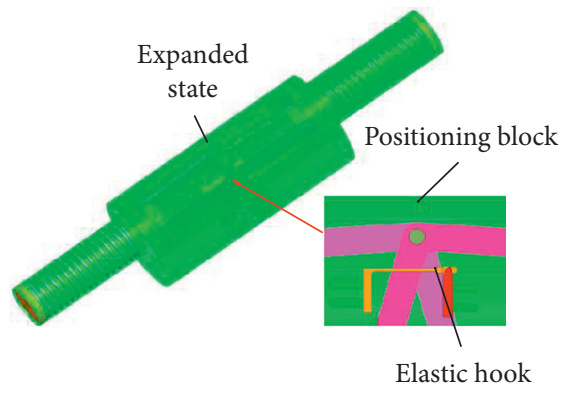

(b)

FIgURE 7: Schematic diagram of the folding hinge deployment process: (a) deployment process; (b) deployment in place locking.

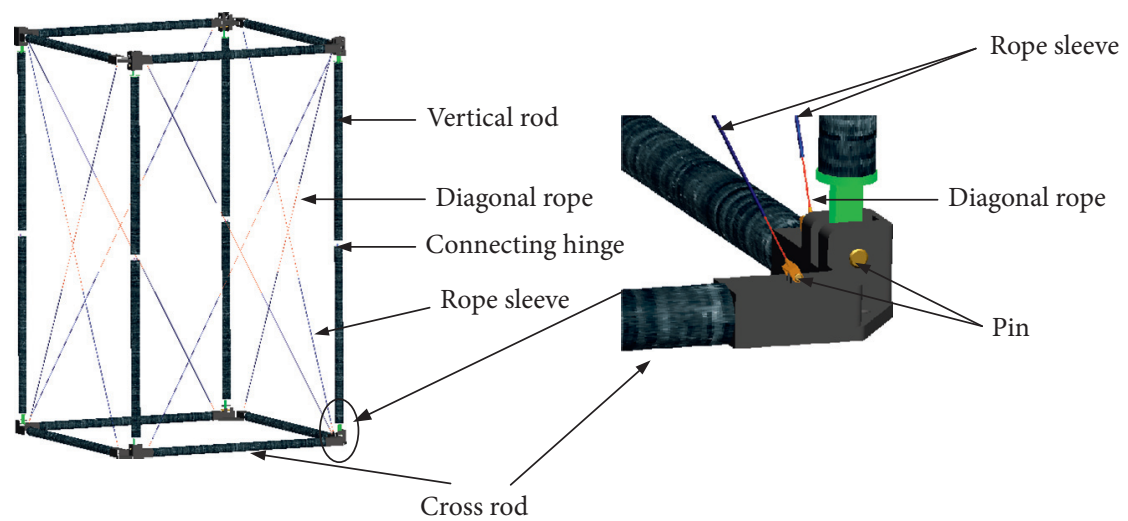

FIgURE 8: Detailed structural design drawing of the expansion unit.

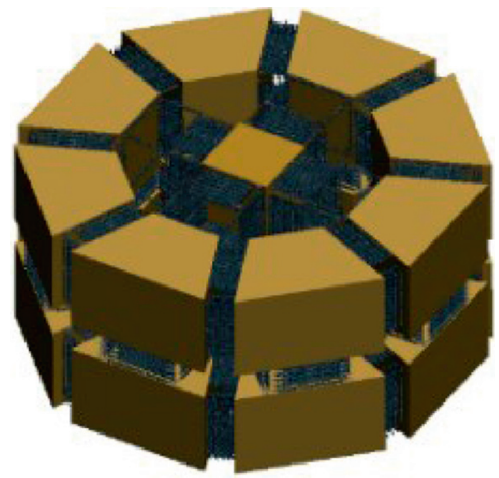

(a)

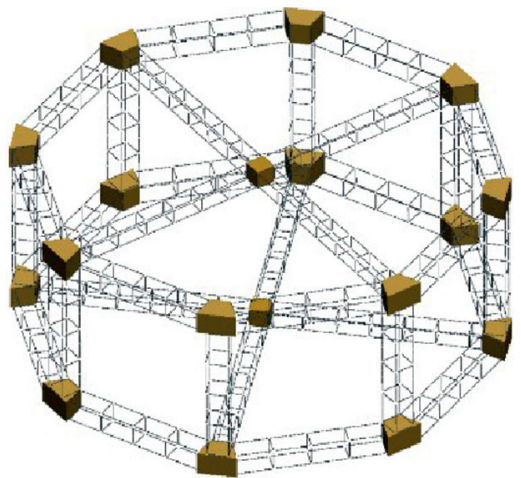

(b)

Figure 9: Overall structural design of the cabin.

height is $2.3 \mathrm{~m}$, whereas the envelope diameter in the unfolded state is $23 \mathrm{~m}$ and the height is $8.4 \mathrm{~m}$.

\section{Establishment of an Equivalent Mechanical Model of the Deployable Cabin System}

\subsection{Establishment of an Equivalent Model of a Continuous Beam with Deployment Element}

3.1.1. Calculation of Strain and Kinetic Energies of the Deployment Unit. The establishment of the continuous beam equivalent model of the deployment unit mechanism can directly obtain the analytical solution of the mechanical characteristics of the system. This model can conveniently evaluate the overall performance of the entire mechanism and facilitate the evaluation when the machine adopts different materials, including the influence of structural parameters on the overall characteristics of the mechanism.

For the convenience of analysis, such as shown in Figure 10, the coordinate system of the rod and the diagonal rope in the deployment unit mechanism and the coordinate system of the basic unit are established, respectively. 


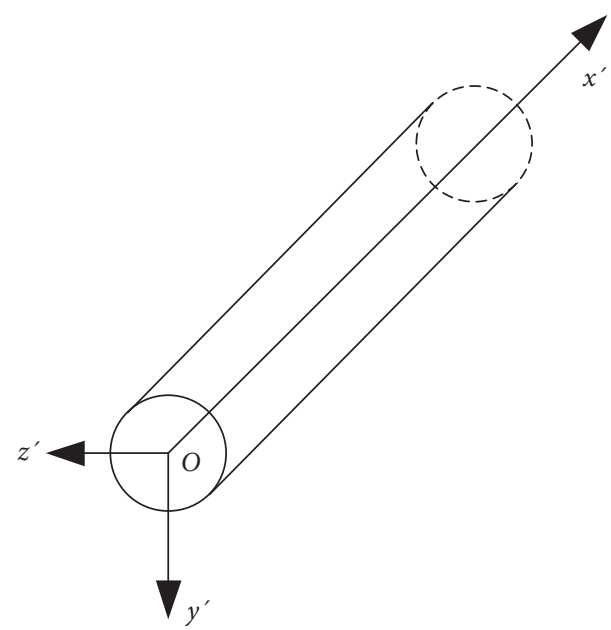

(a)

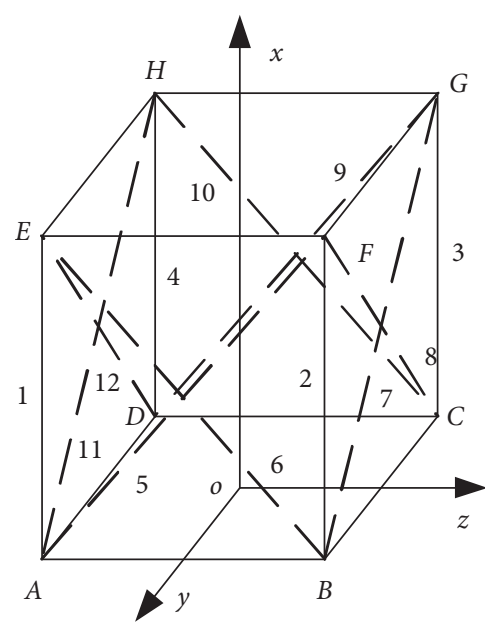

(b)

FIGURE 10: Establishment of the cable member coordinate system.

Let the length of the longitudinal rod of the deployment unit be $l_{a}$, the length of the crossbar is set $l_{b}, l_{c}$, the corresponding side of the cable is $s_{1}, s_{2}$, the included angles between the diagonal rope and the crossbar are, respectively, $\alpha, \beta$, and the lengths of diagonal ropes are $l_{s 1}, l_{s 2}$. According to reference [21], the derivation process will not be repeated, and the strain energy expression of the expansion unit will be directly given. With the central axis strain $\varepsilon_{x}^{0}, k_{x}^{0}, k_{y}^{0}, k_{z}^{0}, \gamma_{x y}^{0}, \gamma_{x z}^{0}$, the expression of the total strain energy of the expressed cell is

$$
\begin{aligned}
U_{\varepsilon}= & \frac{1}{2} \sum E^{(k)} A^{(k)} l^{(k)}\left(\varepsilon^{(k)}\right)^{2} \\
= & {\left[2 E_{a} A_{a} l_{a}+2 E_{s} A_{s}\left(l_{s 1} \sin ^{4} \alpha+l_{s 1} \sin ^{4} \beta\right)\right]\left(\varepsilon_{x}^{0}\right)^{2} } \\
& +\frac{1}{2}\left[E_{s} A_{s} l_{a}\left(l_{s 1} l_{b}^{2} \sin ^{2} \alpha \cos ^{2} \alpha+l_{s 2} l_{c}^{2} \sin ^{2} \beta \cos ^{2} \beta\right)\right]\left(k_{x}^{0}\right)^{2} \\
& +\frac{1}{2}\left[E_{a} A_{a} l_{a}+\frac{1}{2} E_{s} A_{s}\left(l_{s 1} l_{b}^{2} \sin ^{4} \alpha+l_{s 2} l_{c}^{2} \sin ^{4} \beta\right)\right]\left(k_{y}^{0}\right)^{2} \\
& +\frac{1}{2}\left[E_{a} A_{a} l_{a}+\frac{1}{2} E_{s} A_{s}\left(l_{s 1} l_{b}^{2} \sin ^{4} \alpha+l_{s 2} l_{c}^{2} \sin ^{4} \beta\right)\right]\left(k_{z}^{0}\right)^{2} \\
& +l_{b}^{2}\left[E_{s} A_{s} l_{a}\left(l_{s 1} \sin ^{2} \alpha \cos ^{2} \alpha+l_{s 1} \sin ^{2} \beta \cos ^{2} \beta\right)\right]\left(\gamma_{x z}^{0}\right)^{2} \\
& +\left[E_{s} A_{s} l_{a}\left(l_{s 1} \sin ^{2} \alpha \cos ^{2} \alpha+l_{s 1} \sin ^{2} \beta \cos ^{2} \beta\right)\right]\left(\gamma_{x y}^{0}\right)^{2},
\end{aligned}
$$

where $E_{a}, A_{a}, l_{a}$ are the elastic modulus, cross-sectional area, and length of the longitudinal rod, and $E_{s}, A_{s}$ are the elastic modulus and cross-sectional area of the cable, respectively. Then, $\varphi_{x}, \varphi_{y}, \varphi_{z}$ is the corner.

The strain energy of the expandable unit can be expressed as

$$
U_{\varepsilon}=\frac{1}{2} \rho^{T} E \rho,
$$

where

$$
\begin{aligned}
& E=\left[\begin{array}{cccccc}
4 E_{11} & & & & & \\
& E_{22} & & & 0 & \\
& & E_{33} & & & \\
& & & E_{44} & & \\
& 0 & & & 4 E_{55} & \\
& & & & & 4 E_{66}
\end{array}\right]_{6 \times 6} \text {, } \\
& \rho=\left[\varepsilon_{x}^{0}, k_{x}^{0}, k_{y}^{0}, k_{z}^{0}, \gamma_{x y}^{0}, \gamma_{x z}^{0}\right]^{T}, \\
& E_{11}=E_{a} A_{a} l_{a}+E_{s} A_{s} l_{a}\left(l_{s 1} \sin ^{4} \alpha+l_{s 1} \sin ^{4} \beta\right), \\
& E_{22}=E_{s} A_{s}\left(l_{s 1} l_{b}^{2} \sin ^{2} \alpha \cos ^{2} \alpha+l_{s 2} l_{c}^{2} \sin ^{2} \beta \cos ^{2} \beta\right) \text {, } \\
& E_{33}=E_{a} A_{a} l_{a}+E_{s} A_{s}\left(l_{s 1} l_{b}^{2} \sin ^{4} \alpha+l_{s 2} l_{c}^{2} \sin ^{4} \beta\right), \\
& E_{44}=E_{a} A_{a} l_{a}+E_{s} A_{s}\left(l_{s 1} l_{b}^{2} \sin ^{4} \alpha+l_{s 2} l_{c}^{2} \sin ^{4} \beta\right) \text {, } \\
& E_{55}=E_{s} A_{s} l_{a}\left(l_{s 1} \sin ^{2} \alpha \cos ^{2} \alpha+l_{s 2} \sin ^{2} \beta \cos ^{2} \beta\right) \text {, } \\
& E_{66}=E_{s} A_{s} l_{a}\left(l_{s 1} \sin ^{2} \alpha \cos ^{2} \alpha+l_{s 2} \sin ^{2} \beta \cos ^{2} \beta\right) \text {. }
\end{aligned}
$$

The rigid body motion of the unfolding unit is the main part of the kinetic energy. To simplify the analysis, the strain term in the displacement is ignored when calculating the kinetic energy of the unfolding unit. According to reference 24 , the derivation process will not be repeated, and the kinetic energy expression of the expansion unit will be directly given:

$$
\begin{aligned}
E_{K}= & 2\left[\rho_{a} A_{a} l_{a}+\frac{1}{2} \rho_{b} A_{b}\left(l_{b}+l_{c}\right)+\rho_{s} A_{s}\left(l_{s 1}+l_{s 2}\right)\right. \\
& \left.+m_{1}+m_{2}\right] \omega^{2}\left(u^{0} u^{0}+v^{0} v^{0}+w^{0} w^{0}\right) \\
& +\left[l_{b}^{2}\left(\frac{1}{2} \rho_{a} A_{a} l_{a}+\frac{1}{6} \rho_{b} A_{b} l_{b}+\frac{1}{3} \rho_{s} A_{s} l_{s 1}\right)\right. \\
& \left.+l_{c}^{2}\left(\frac{1}{2} \rho_{a} A_{a} l_{a}+\frac{1}{6} \rho_{b} A_{b} l_{c}+\frac{1}{3} \rho_{s} A_{s} l_{s 2}\right)\right] \omega^{2}\left(\varphi_{x} \varphi_{x}+\varphi_{y} \varphi_{y}\right) \\
& +\left[l_{b}^{2}\left(\frac{1}{2} \rho_{a} A_{a} l_{a}+\frac{1}{6} \rho_{b} A_{b} l_{b}+\frac{1}{3} \rho_{s} A_{s} l_{s 1}\right)\right. \\
& \left.+l_{c}^{2}\left(\frac{1}{2} \rho_{a} A_{a} l_{a}+\frac{1}{6} \rho_{b} A_{b} l_{c}+\frac{1}{3} \rho_{s} A_{s} l_{s 2}\right)\right] \omega^{2}\left(\varphi_{x} \varphi_{x}+\varphi_{z} \varphi_{z}\right),
\end{aligned}
$$


where $\rho_{a}, \rho_{b}, \rho_{s 1, s 2}$ pertain to the density of longitudinal bar, transverse bar, and cable, respectively. Then, $u^{0}, v^{0}, w^{0}$ and $y=z=0$ are the displacement at the position, that is, the axial displacement of the unfolded unit. $m_{1}, m_{2}$, respectively, are the quality of joints and corner blocks, and $\omega$ is the vibration circle frequency of the unfolded unit. In $\delta=\left(u^{0}, v^{0}, w^{0}, \varphi_{x}, \varphi_{y}, \varphi_{z}\right)^{T}$, the vector of the total kinetic energy of the expansion unit is expressed as

$$
E_{K}=\frac{1}{2} \omega^{2} \delta^{T} N \delta
$$

where

$$
\begin{aligned}
& N=2\left[\begin{array}{cccccc}
2 M_{11} & & & & & \\
& 2 M_{11} & & & 0 & \\
& & 2 M_{11} & & & \\
& & & 2 M_{22} & & \\
& & & & M_{22} & \\
& & & & & M_{22}
\end{array}\right]_{6 \times 6} \\
& M_{11}=2\left[\rho_{a} A_{a} l_{a}+\frac{1}{2} \rho_{b} A_{b}\left(l_{b}+l_{c}\right)+\rho_{s} A_{s}\left(l_{s 1}+l_{s 2}\right)+m_{1}+m_{2}\right], \\
& M_{22}=\left[l_{b}^{2}\left(\frac{1}{2} \rho_{a} A_{a} l_{a}+\frac{1}{6} \rho_{b} A_{b} l_{b}+\frac{1}{3} \rho_{s} A_{s} l_{s 1}\right)\right. \\
& \left.+l_{c}^{2}\left(\frac{1}{2} \rho_{a} A_{a} l_{a}+\frac{1}{6} \rho_{b} A_{b} l_{c}+\frac{1}{3} \rho_{s} A_{s} l_{s 2}\right)\right] \text {. }
\end{aligned}
$$

3.1.2. Establishment of the Equivalent Beam Model. According to the principle of equal strain energy, the strain energy of a certain length of the continuous beam is equal to that of the deployment element. Combining the expression of strain and kinetic energies of the deployment element equations (1)-(5), the equivalent stiffness expression of the equivalent model of continuous beam obtained by calculation is

$$
\left\{\begin{array}{l}
E I_{y}=2\left(E_{a} A_{a}+E_{s} A_{s} \sin ^{3} \alpha\right) R^{2} \\
E I_{z}=2\left(E_{a} A_{a}+E_{s} A_{s} \sin ^{3} \beta\right) R^{2} \\
G J=2 E_{s} A_{s} R^{2}\left(\sin \alpha \cos ^{2} \alpha+\sin \beta \cos ^{2} \beta\right) \\
G A_{y}=4 E_{s} A_{s} \sin \alpha \cos ^{2} \alpha \\
G A_{z}=4 E_{s} A_{s} \sin \beta \cos ^{2} \beta \\
E A=4 E_{a} A_{a}+4 E_{s} A_{s}\left(\sin ^{3} \alpha+\sin ^{3} \beta\right)
\end{array}\right.
$$

where $E I_{y}, E I_{z}$ are bending stiffness of equivalent beam relative to $Y$ axis and $Z$ axis, and $G A_{y}, G A_{z}$ are the shear stiffness of equivalent beam along the $y$ and $z$ axes, respectively. $G J$ is the equivalent beam torsional stiffness, and $E A$ is the axial stiffness.

According to the conservation of kinetic energy, the kinetic energy of the continuous beam is equal to that of the unfolded element. Moreover, the equivalent mass parameters of the equivalent model of the continuous beam are obtained as follows:

$$
\left\{\begin{array}{l}
m_{11}=4\left[\rho_{a} A_{a}+\frac{1}{2} \rho_{b} A_{b}\left(\frac{l_{b 1}}{l_{a}}+\frac{l_{b 2}}{l_{a}}\right) 2+\frac{1}{2} \rho_{s} A_{s}\left(\frac{l_{s 1}}{l_{a}}+\frac{l_{s 2}}{l_{a}}\right)+\frac{m_{1}}{l_{a}}+\frac{m_{2}}{l_{a}}\right], \\
m_{12}=m_{13}=m_{23}=0, \\
m_{22}=\left(\frac{1}{2} \rho_{a} A_{a}+\frac{1}{3} \rho_{b} A_{b} \frac{l_{b 1}}{l_{a}}+\frac{2}{3} \rho_{s} A_{s} \frac{l_{s 1}}{l_{a}}+\frac{1}{2} \frac{m_{1}}{l_{a}}+\frac{1}{2} \frac{m_{2}}{l_{a}}\right) l_{b 1}^{2}+\left(\frac{1}{2} \rho_{a} A_{a}+\frac{1}{3} \rho_{b} A_{b} \frac{l_{b 2}}{l_{a}}+\frac{2}{3} \rho_{s} A_{s} \frac{l_{s 2}}{l_{a}}+\frac{1}{2} \frac{m_{1}}{l_{a}}+\frac{1}{2} \frac{m_{2}}{l_{a}}\right) l_{b 2}^{2}, \\
m_{33}=\left(\frac{1}{2} \rho_{a} A_{a}+\frac{1}{3} \rho_{b} A_{b} \frac{l_{b 1}}{l_{a}}+\frac{2}{3} \rho_{s} A_{s} \frac{l_{s 1}}{l_{a}}+\frac{1}{2} \frac{m_{1}}{l_{a}}+\frac{1}{2} \frac{m_{2}}{l_{a}}\right) l_{b 1}^{2}+\left(\frac{1}{2} \rho_{a} A_{a}+\frac{1}{3} \rho_{b} A_{b} \frac{l_{b 2}}{l_{a}}+\frac{2}{3} \rho_{s} A_{s} \frac{l_{s 2}}{l_{a}}+\frac{1}{2} \frac{m_{1}}{l_{a}}+\frac{1}{2} \frac{m_{2}}{l_{a}}\right) l_{b 2}^{2},
\end{array}\right.
$$

where $m_{11}$ is the equivalent beamline density, and $m_{22}, m_{33}$ are the equivalent beam moment of inertia coefficient.

\subsection{Construction of the Equivalent Static Model of the De-} ployable Cabin System and Verification. Based on the equivalent beam model of the deployment element established above, each equivalent beam model is assembled according to the topological relation shown in Figure 8 . Thus, the equivalent mechanical model of the entire deployment cabin system is obtained, as shown in Figure 11.
The statics characteristic characterizes the ability of the entire system to resist loads. The cabin system will be subjected to various forms of space loads during its orbiting operation. Therefore, the main mechanical characteristic parameters of the static stiffness and strength of the system should be analyzed and deduced, which mainly include the following: axial tension, lateral bending, and torsion around the optical axis. The deployable compartment mechanism is a quasistatic structure after being fully deployed, which has the 


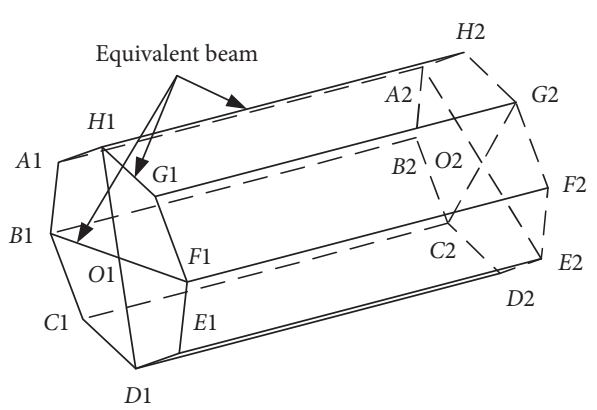

Figure 11: Equivalent model of the deployable cabin system.

characteristics of large flexibility and large size. Therefore, studying the dynamic characteristics of the deployable cabin section in its deployment state is of great significance.

\subsubsection{Theoretical Calculation of Static Characteristics of the Equivalent Model of the Cabin}

(1) Axial Tensile Stiffness. The tensile stiffness of the expanded element equivalent beam model is expressed as $E A$, and the tensile stiffness of the whole cabin system is expressed as $E_{l} A_{l}$, combined with the equivalent model of the system. Then, the axial tensile stiffness of the system can be deduced as follows:

$$
E_{l} A_{l}=8 E A .
$$

When the system is subjected to axial tension, the deformation of the support truss mechanism at both ends of the cabin system will also affect its tensile stiffness, and its influence also needs to be considered.

As shown in Figure 12, the total axial length of the deployable cabin system $L_{l}$ is set, and the cabin end support truss is subjected to axial tension $P$ under the action of axial tension. Moreover, the axial deformation of the end support truss of the cabin system can be expressed as $\Delta_{l}=\left(2 P L_{l} / E_{l} A_{l}\right)$, and the total axial deformation of the cabin system under the action of axial tension $P$ is

$$
\Delta_{z}=\Delta_{L}+\Delta_{l}=\frac{P L}{8 E A}+\frac{2 P L_{l}}{E_{l} A_{l}}=\frac{P\left(L+2 L_{l}\right)}{8 E A} .
$$

Through the definition of tensile stiffness, the equivalent stiffness relationship between the axial tensile stiffness of the cabin system, the supporting truss mechanism at both ends of the cabin system, and the axially unfolded structure can be deduced as follows:

$$
E_{Z} A_{Z}=\frac{8\left(L+2 L_{l}\right) E A \cdot E_{l} A_{l}}{L E_{l} A_{l}+16 L_{l} E A} .
$$

(2) Bending Stiffness. The cabin system mainly includes twoend supporting trusses and eight axially extending trusses. When analyzing the bending stiffness of the system, the geometric dimensions of the two-end supporting trusses are very small compared with the eight axially extending truss

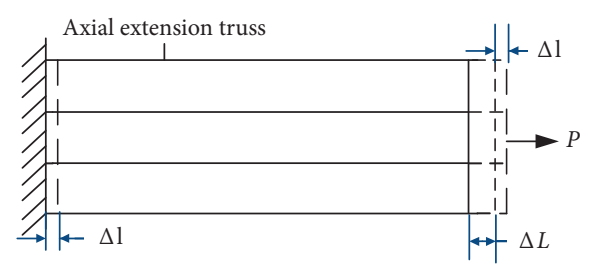

Figure 12: Axial bearing deformation diagram of the system.

mechanisms, and the bending stiffness is relatively large. In the process of analyzing the bending stiffness of the cabin system, the influence of this part can be ignored. For the entire cabin system, analyzing its bending stiffness can be regarded as analyzing the bending stiffness of octagonal space structure surrounded by equivalent continuous beams of eight trusses in the axial direction of the cabin system.

The moment of inertia of the system is initially calculated. Figure 13 depicts the cross-sectional view of the cabin system, establishing a rectangular coordinate system. The $x$ and $y$ axes are perpendicular to the edge of the octagonal prism. In the xoy plane, the radius of the circumscribed circle of the octagonal prism is defined as $R$, the azimuth of the equivalent axial support truss is $\theta$, and then, the azimuth of the eight equivalent axial trusses is

$$
\theta_{i}=\theta_{1}+\frac{\pi}{4} i, \quad(i=1 \sim 7),
$$

where $\theta_{1}$ is the azimuth of the first equivalent axial support truss.

The distance from the equivalent support truss to the $y$ axis is

$$
x_{i}=R \cos \theta_{i} .
$$

The moment of inertia of the cabin system to the $y$ axis is

$$
I_{y}=8 I+\sum_{i=1}^{7} A\left(x_{i}\right)^{2}=8 I+A R^{2} \sum_{i=1}^{7} \cos ^{2}\left(\theta_{1}+\frac{\pi}{4} i\right)=8 I+4 A R^{2} .
$$

Among them, $I$ is the equivalent beam moment of inertia, and $A$ is the equivalent beam area:

$$
I=\frac{l^{4}}{12}=\frac{A^{2}}{12}
$$

To sum up, the moment of inertia of the cabin system to the $y$ axis is

$$
I_{y}=\frac{A^{2}}{3}+4 A R^{2} .
$$

Ignoring the influence of the upper and lower bottom support trusses, the bending stiffness of the cabin system is

$$
E_{Z} I_{Z}=E\left(\frac{A^{2}}{3}+4 A R^{2}\right) \text {. }
$$

(3) Torsional Stiffness Calculation. The deployable cabin system is subject to a moment $M$. The single-root 


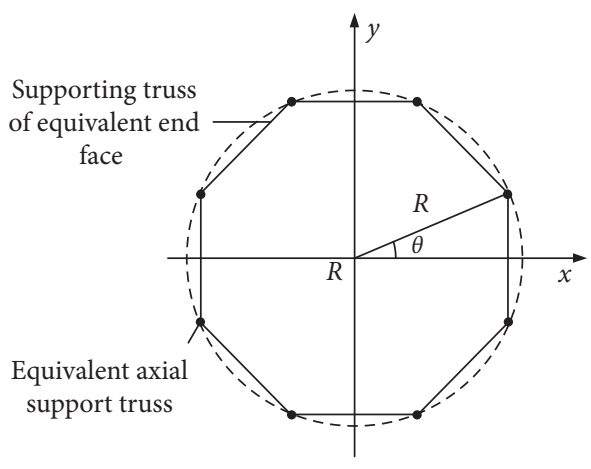

FIGURE 13: Section of the cabin system.

longitudinal equivalent beam has been not only reversed but also bent. Such as shown in Figure 14, the torque applied to the system when the system is reversed $M$ is equivalent to the upper bottom of eight stretching mechanisms. Each longitudinal equivalent beam receives a force, respectively, $F$ and moment $M^{\prime}$, the torque can be obtained as follows:

$$
M=4 F R+8 M^{\prime},
$$

where $M$ is the torque of the deployable cabin system, $\mathrm{F}$ is the longitudinal equivalent beam of the equal effect of the end, and $M^{\prime}$ is the longitudinal equivalent beam of equivalent moment in the end.

When the system receives torque $M$, longitudinal equivalent beam of the end twist angle $d \theta_{n}$ and system end twist angle $\Delta \theta_{n}$, and longitudinal equivalent beam bending angle of the end $d \theta_{w}$, the relationship with the corner at the end of the system is

$$
\Delta \theta_{w}=2 \arcsin \left(R \sin \frac{\Delta \theta_{n}}{2 L}\right) .
$$

The relationship between the torsion angle of the system and the external force is

$$
\Delta \theta_{n}=\frac{M L_{l}}{G_{Z} J_{Z}}
$$

where $G_{Z} J_{Z}$ is the equivalent torsional stiffness of the system. When $M$ is equivalent to the longitudinal equivalent beam, the resulting bending and torsion angles are

$$
\begin{aligned}
& \Delta \theta_{w}=\frac{F L^{2}}{2 E I}, \\
& \Delta \theta_{n}=\frac{M^{\prime} L}{G J} .
\end{aligned}
$$

Eight equivalents beam the equivalent torsional stiffness of the parallel connection as follows:

$$
G_{8} J_{8}=8 D^{2} E \frac{I}{L^{2}}+8 G J .
$$

At the moment of octagonal top and bottom supporting truss $M$, the resulting corner is

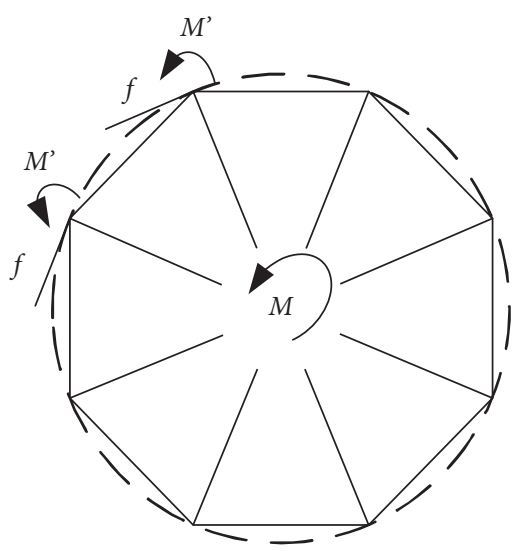

FIGURE 14: System torsion deformation diagram.

$$
\Delta \theta_{n 1}=\frac{2 M l}{G_{l} J_{l}}
$$

The equivalent torsional stiffness of the deployable cabin system is

$$
\frac{1}{G_{Z} J_{Z}}=\frac{L^{3}}{8(L+2 l)\left(D^{2} E I+L^{2} G_{8} J_{8}\right)}+\frac{2 l}{(L+2 l) G_{l} J_{l}} .
$$

By combining the calculation formula of the equivalent beam coefficient in Section 3.1 and the structural parameters of the deployable cabin system, the statics parameters of the deployable cabin system can be obtained.

3.2.2. Verification of the Static Equivalent Model. According to the equivalent parameters of the continuous beam obtained in Section 3.1, combined with the structural and material parameters of the deployable element, the equivalent beam model parameters of the cabin segment deployable element can be calculated. According to the calculation that the envelope diameter of the expandable cabin is $23 \mathrm{~m}$ and the height is $8 \mathrm{~m}$, Table 3 shows the parameter of the material used by the expandable unit. Then, Table 4 shows the structural parameter of the expandable unit.

Carbon fiber is used for the rod material of the deployable unit of the deployable cabin system. Kevlar is used for the cable, and the specific parameters are as follows. The corner block uses an aluminum alloy material. The quality of the corner block is $m_{1}=0.4 \mathrm{Kg}, m_{2}=0.3 \mathrm{Kg}$. Then, the parameters are substituted into equations (7) and (8), and Table 5 presents the list of the equivalent beam model parameters.

According to the derivation of static parameters, such as tensile stiffness, bending stiffness, and torsional stiffness, of the deployable cabin section in Section 3.2.1, combined with the calculation results of equivalent beam model parameters, the static parameters of the entire cabin section system can be calculated. Table 6 shows the list of the equivalent static parameters of the cabin system.

To verify the correctness of the calculation of the static parameters of the system, the finite element model of the cabin system is established in the finite element 
TABLE 3: Material parameters of expandable units.

\begin{tabular}{lccc}
\hline Material & Density $\left(\mathrm{kg} / \mathrm{m}^{3}\right)$ & Modulus of elasticity $(\mathrm{GPa})$ & Poisson's ratio \\
\hline Kevlar49 fiber & 1,450 & 131 & 0.30 \\
Aluminum alloy & 2,840 & 70 & 0.31 \\
Titanium alloy & 4,510 & 110 & 0.34 \\
Carbon fiber & 1,740 & 230 & 0.31 \\
\hline
\end{tabular}

TABLE 4: Structural parameters of expandable units.

\begin{tabular}{lccc}
\hline Parameter name & Numerical value & Parameter name & Numerical value \\
\hline Cross bar diameter $(\mathrm{mm})$ & $28 / 26$ & Bar density $\left(\mathrm{kg} / \mathrm{m}^{3}\right)$ & 1,740 \\
Diameter of longitudinal rod $(\mathrm{mm})$ & $30 / 28$ & Cable diameter $(\mathrm{mm})$ & 1 \\
Cross bar length $l_{b 1, b 2}(\mathrm{~mm})$ & 900 & Elastic modulus $(\mathrm{GPa})$ of cable & 131 \\
Longitudinal rod length $l_{\mathrm{A}}(\mathrm{mm})$ & 1200 & Angle $\alpha$ (degrees) & 53.13 \\
\hline
\end{tabular}

TABLE 5: Equivalent beam model parameters.

\begin{tabular}{|c|c|c|c|c|}
\hline $\begin{array}{l}\text { Bending stiffness } \\
E I\left(N \times m^{2}\right)\end{array}$ & $\begin{array}{l}\text { Torsional stiffness } \\
\quad G J(N \times m)\end{array}$ & $\begin{array}{l}\text { Shear stiffness } \\
G A(\mathrm{~N})\end{array}$ & Axial stiffness $E A(\mathrm{~N})$ & Unit line density $m_{11}(\mathrm{Kg} / \mathrm{m})$ \\
\hline $6.81 \times 10^{7}$ & $1.92 \times 10^{7}$ & $1.19 \times 10^{7}$ & $1.26 \times 10^{8}$ & 1.79 \\
\hline
\end{tabular}

TABLE 6: Equivalent static parameters of the cabin system.

\begin{tabular}{lcr}
\hline Axial stiffness $E_{Z} A_{Z}(\mathrm{~N})$ & Bending stiffness $E_{Z} I_{Z}\left(N \times m^{2}\right)$ & Torsional stiffness $G_{Z} J_{Z}(N \times m)$ \\
\hline $2.28 \times 10^{8}$ & $5.04 \times 10^{9}$ & $3.84 \times 10^{7}$ \\
\hline
\end{tabular}

software. The corresponding load is applied, and the deformation of the cabin system under the applied load is measured. Then, the static parameters of the expandable cabin system are calculated using the mechanical formulas.

When building a model of the cabin system in the software, the parametric modeling function of the software is used. When assigning cross-sectional attributes, beam elements are used for the structure of the extension mechanism, such as the rods and propulsion elements. Moreover, the cross-sectional shapes are loops and rectangles, respectively. For diagonal ropes, truss elements that do not consider compressive stiffness are used for simulation. In the finite element simulation analysis, a rope can only be divided into one element, and the rope crossing in the model cannot be broken. If it is divided into multiple elements or there is disconnection at the intersection, the stiffness of the system will be reduced. First, an axial tensile stiffness simulation of the cabin system is conducted. Moreover, a pulling force of $1 \mathrm{KN}$ is applied to the upper and lower surfaces of the deployable cabin system along the optical axis of the finite element model of the cabin system, through simulation. The axial deformation of the deployable cabin system is $3.8617 \times 10^{-5} \mathrm{~m}$.

The axial tensile stiffness of the cabin system calculated according to the formula is

$$
E_{Z} A_{Z}=\frac{F l}{\Delta l}=\frac{1.0 \times 10^{3} \times 8}{3.8617 \times 10^{-5}}=2.0716 \times 10^{8} \mathrm{~N}
$$

Second, the bending stiffness of the cabin system is simulated, and the force along the horizontal direction is added at its central position, with a magnitude of $50 \mathrm{~N}$. Through the simulation results, the deformation of the system is $7.1038 \times 10^{-7} \mathrm{~m}$. According to the mechanical formula, the bending stiffness of the system can be calculated as follows:

$$
E_{Z} I_{Z}=-\frac{F L^{3}}{8 v}=\frac{50 \times 8^{3}}{8 \times 7.1038 \times 10^{-7}}=4.5046 \times 10^{9} \mathrm{~N} \times \mathrm{m}^{2}
$$

Finally, the torsional stiffness of the cabin system is simulated, and the applied size on the deployable cabin system is $5 \mathrm{~N} \cdot \mathrm{m}$. The direction of the torque is approximately at the $z$ axis of the deployable cabin system. Through the finite element simulation results, the torsion angle of the cabin system around the $z$ axis is $1.0887 \times 10^{-6}$ degree. According to the torsion angle equation of the deployable cabin system under torque, the calculated torsional stiffness is

$$
G_{Z} I_{Z}=\frac{T L}{\phi}=\frac{5 \times 8}{1.0887 \times 10^{-6}}=3.6741 \times 10^{7} \mathrm{~N} \cdot \mathrm{m}^{2} .
$$

Comparing the theoretical calculation results of the axial tensile, bending, and torsional stiffness of the equivalent model of the deployable cabin system with the finite element simulation results, the errors are $10.06 \%, 11.89 \%$, and $4.52 \%$, which are shown in Figure 15. Results show that the statics model of the system equivalent model has high credibility. 


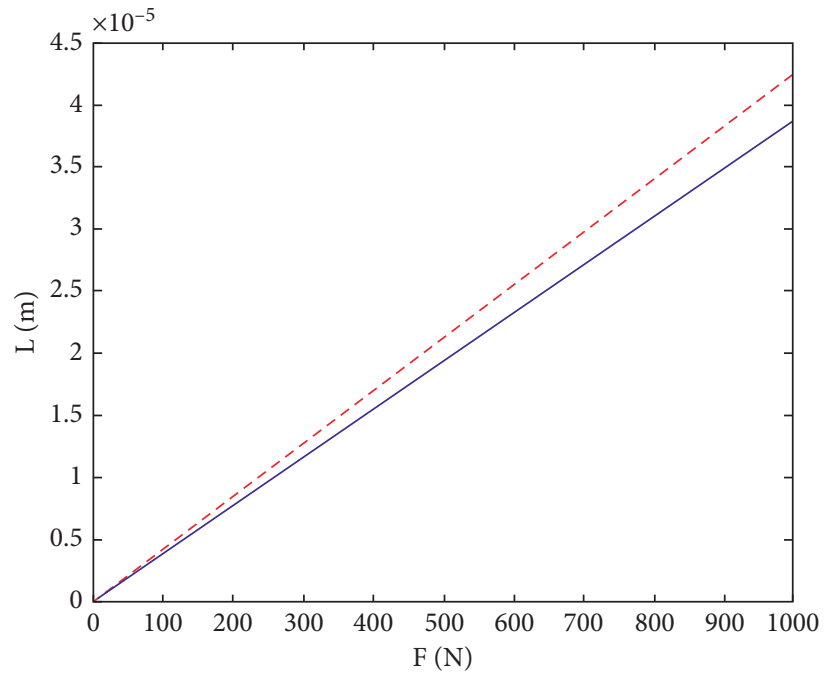

_ Equivalent stiffness

_. _ Finite element stiffness

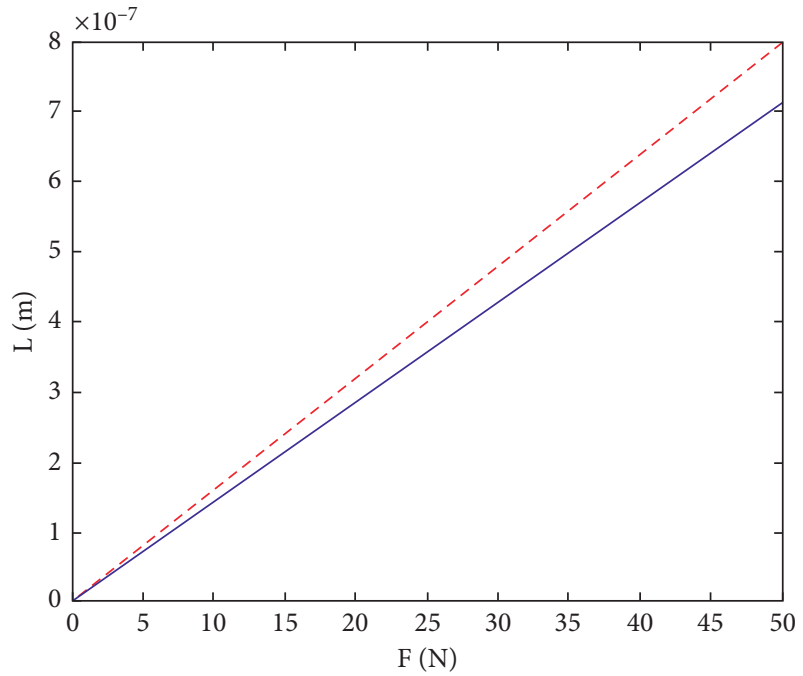

Equivalent stiffness

_. - Finite element stiffness

(a)

(b)

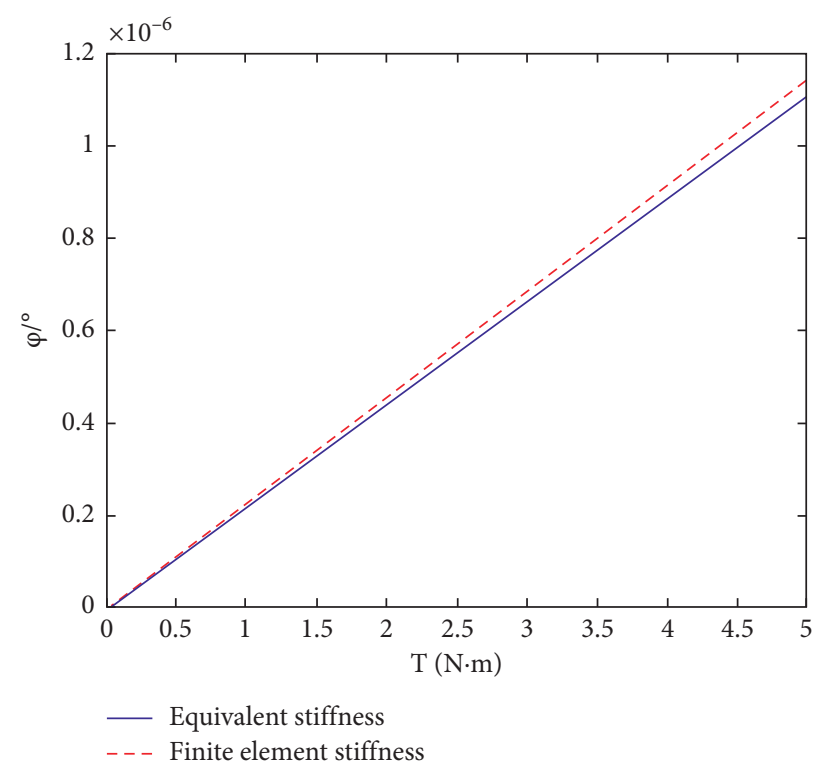

(c)

FIGURE 15: Stiffness error comparison: (a) axial stiffness, (b) bending stiffness, and (c) torsional stiffness.

3.3. Construction and Verification of an Equivalent Dynamic Model of a Deployable Cabin System. Based on the equivalent mechanical model of the cabin system established in Section 3.2, the vibration frequency and corresponding vibration mode of the sixth-order fixed bottom before the equivalent dynamic of the system are calculated by using the relevant formula of vibration mechanics. To verify the correctness of the theoretical calculation, the finite element model established in Section 3.2.2 is also calculated in this study. Figure 16 depicts the comparison of the first six vibration modes.

As shown in Figure 16, the theoretical calculation results of the equivalent model are similar to the finite element simulation results, which proves the correctness of the theoretical analysis.

Table 7 shows the first six modal frequency pairs obtained by theoretical analysis and finite element calculation. 


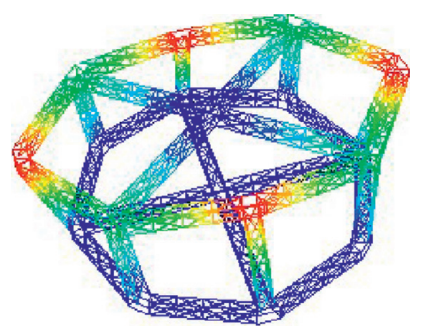

(a)

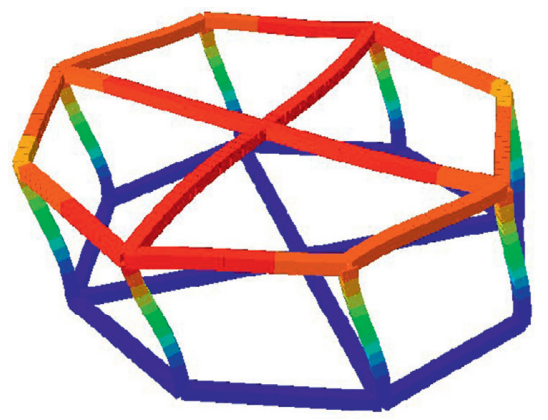

(d)

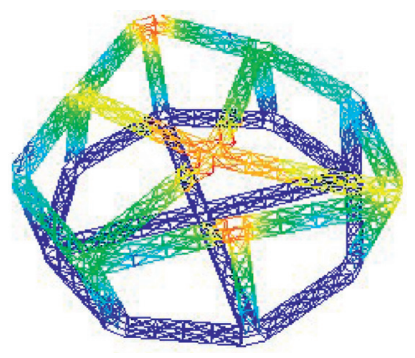

(g)

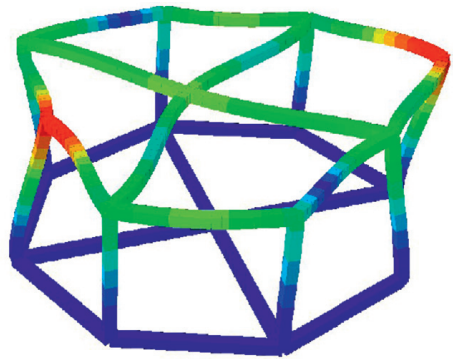

(j)

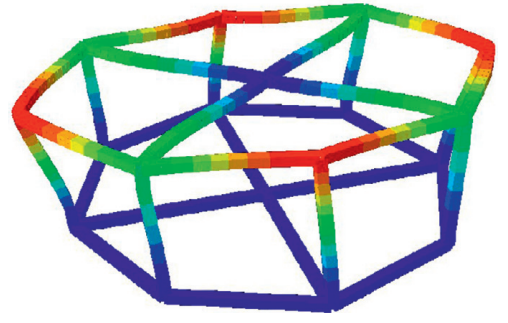

(b)

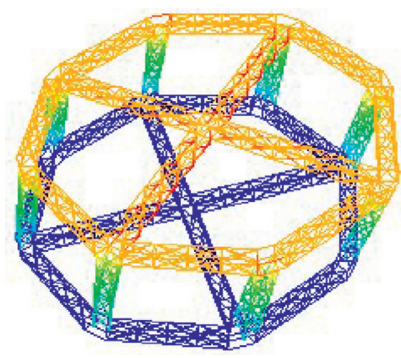

(e)

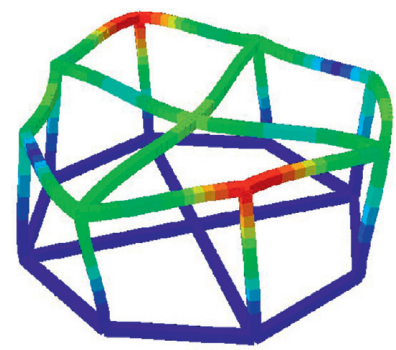

(h)

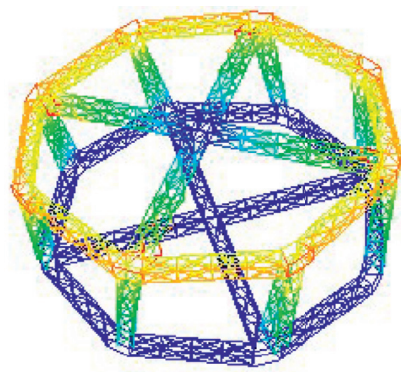

(k)

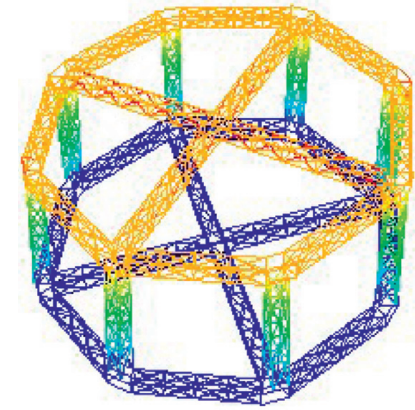

(c)

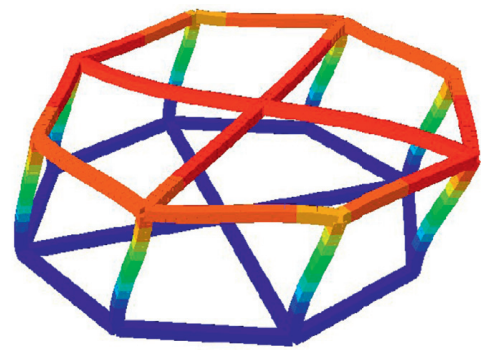

(f)

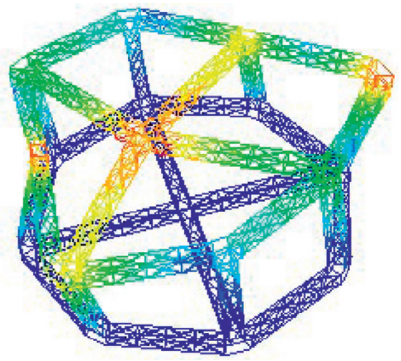

(i)

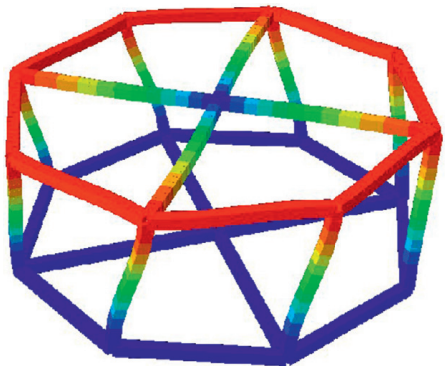

(l)

FIGURE 16: Comparison of the first six vibration modes of the cabin system vibration frequency: (a) finite first-order vibration mode, (b) equivalent model first-order vibration mode, (c) finite element second-order mode, (d) equivalent model second-order mode, (e) finite element third-order mode, (f) equivalent model third-order mode, (g) finite element fourth-order vibration mode, (h) equivalent model fourth-order vibration mode, (i) finite element fifth vibration mode, (j) equivalent model fifth vibration mode, (k) finite element sixth vibration mode, and (l) equivalent model sixth vibration mode.

In Table 7, the theoretical calculation error of the equivalent model is relatively low, which proves the accuracy of the calculation of the dynamic characteristics of the system equivalent model. In addition, the calculation time is reduced from $15.68 \mathrm{~min}$ to $12.53 \mathrm{~s}$, and the calculation efficiency is greatly improved, which lays a foundation for the simplified calculation of system mechanical characteristics.

\section{Development of a Prototype System for the Deployable Cabin}

To verify the rationality of the design scheme and the feasibility of the driving scheme, the 1/4 ring of the expandable cabin section and the principle prototype of the stretching mechanism composed of three units are made. The folding 
TABLE 7: Comparison of the first six natural frequencies of the cabin system.

\begin{tabular}{lccc}
\hline Order & Finite element calculation frequency $(\mathrm{Hz})$ & Equivalent model theory calculation frequency $(\mathrm{Hz})$ & Error $(\%)$ \\
\hline 1 & 0.7568 & 0.8146 & 7.64 \\
2 & 0.7743 & 0.8157 & 5.35 \\
3 & 1.1006 & 0.9636 & 12.45 \\
4 & 1.2457 & 1.1261 & 9.60 \\
5 & 1.3068 & 1.3728 & 5.05 \\
6 & 1.5136 & 1.5838 & 4.64 \\
\hline
\end{tabular}

TABLE 8: Geometric parameters and materials used for prototype parts.

\begin{tabular}{|c|c|c|c|c|c|}
\hline Parameter name & Numerical value & Material & Parameter name & Material & Material \\
\hline Truss crossbar length (mm) & 75 & \multirow{5}{*}{ Carbon fiber Wei } & Hinge envelope diameter $(\mathrm{mm})$ & \multirow{5}{*}{$\Phi 7 / 6$} & Copper alloy \\
\hline Truss vertical bar length (mm) & 74 & & Cross bar diameter $(\mathrm{mm})$ & & Carbon fiber \\
\hline Edge vertical bar length (mm) & 86.5 & & Center connection truss & & Nylon glass fiber \\
\hline Edge bar length (mm) & 80 & & Connection joint & & $\begin{array}{l}\text { High-performance } \\
\text { nylon }\end{array}$ \\
\hline Diameter of vertical rod (mm) & $\Phi 9 / 8$ & & Propulsion unit & & Nylon glass fiber \\
\hline
\end{tabular}

height of the longitudinal stretching mechanism is $0.29 \mathrm{~m}$, the height in the unfolded state is $1 \mathrm{~m}$, the envelope diameter is $0.4 \mathrm{~m}$, and the driving mode of applying turbofan to the end bracket is adopted. All the rod materials are carbon fiber, and the joints are processed by 3D printing. The two prototypes are, respectively, tested on the ground to verify the feasibility of the radial deployment scheme and the driving mode.

4.1. Cabin End Mechanism Scale Prototype Development. The overall system structure of the expandable compartment designed in this study is large in size, with thousands of components. The number of mechanical parts to make a complete scaled model is quite large, and the production process is complicated. The axial expansion method of the expandable compartment is a simple one-dimensional expansion, and its expansion method is easy to verify. On the premise of ensuring correctness, to reduce the workload of making prototypes, a quarter of the prototypes that can be deployed are made. The 30:1 scaled prototype machine model is processed. Table 8 shows the specific geometric parameters and materials used by the unfolding unit to process the prototype components. The joints are all processed by $3 \mathrm{D}$ printing.

Figure 17 shows some 3D printed and metal parts.

Figure 18 shows the folded, intermediate, and unfolded states of the processed and assembled prototype machine. The principle prototype is composed of a horizontal bar, a vertical bar, upper and lower corner blocks, a middle hinge, a middle corner block, and a drive spring. The envelope diameter is $120 \mathrm{~mm}$, the unfolding height is $215 \mathrm{~mm}$, the collapsed height is $25.5 \mathrm{~mm}$, and the axial fold-to-length ratio is 8.43 .

As shown in Figure 19, add a torsion spring to drive the root hinge.

The zero-gravity working environment of the truss is different from the Earth's gravity environment. Thus, simulating the real working environment of the deployable

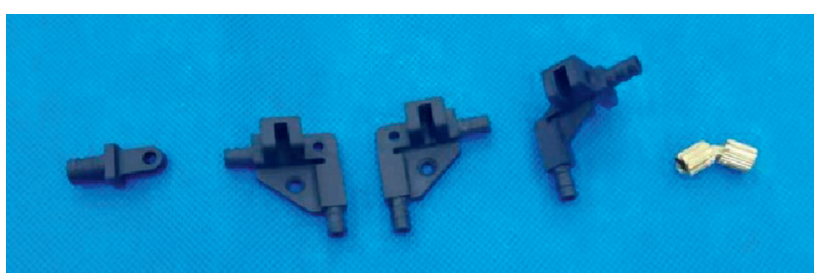

FIGURE 17: Joint, block, block, middle connecting corner block, and hinge.

cabin section truss mechanism in zero gravity in space is necessary. The suspension support method is adopted as the gravity compensation design scheme of the deployable truss. Figure 20 shows the test device built.

Figure 21 shows the unfolding process of the prototype and that the entire mechanism is unfolded smoothly without jamming, which proves the rationality of the structural design of the end face of the system.

4.2. Longitudinal Stretching Mechanism Prototype Development. In this study, an extension mechanism constructed by three units is designed to verify the reliability of the drive, as shown in Figure 22. The drive turbofan and the stretching mechanism fixing brackets are, respectively, installed on the sides of the extension mechanism, and the turbofan is used to simulate the miniature rocket engine. The hinge material is a copper alloy, the rod material is carbon fiber, the joint uses a nylon glass fiber material, and the fixing bracket is an acrylic board. The folding height of the mechanism is $0.29 \mathrm{~m}$, the unfolding height is $1 \mathrm{~m}$, and the envelope diameter is $0.4 \mathrm{~m}$.

In this study, an experimental platform for the unfolding function of the stretching mechanism is built, as shown in Figure 23. The extension mechanism is fixed to the support frame to counteract the influence of gravity. To ensure the accuracy of the experiment, the extension mechanism is suspended. Two sliding blocks are installed on the guide 


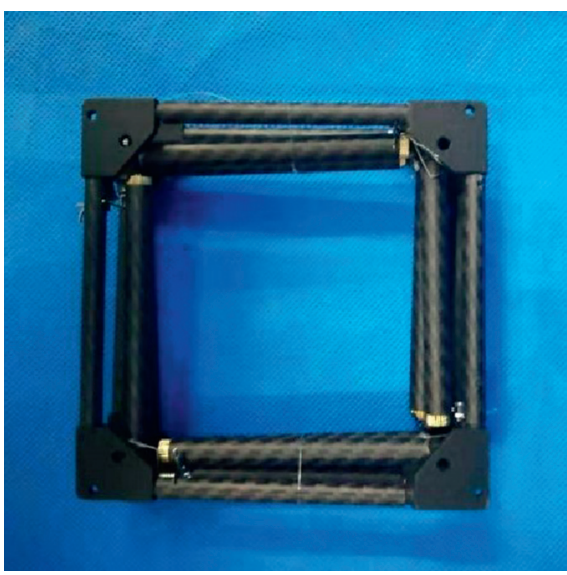

(a)

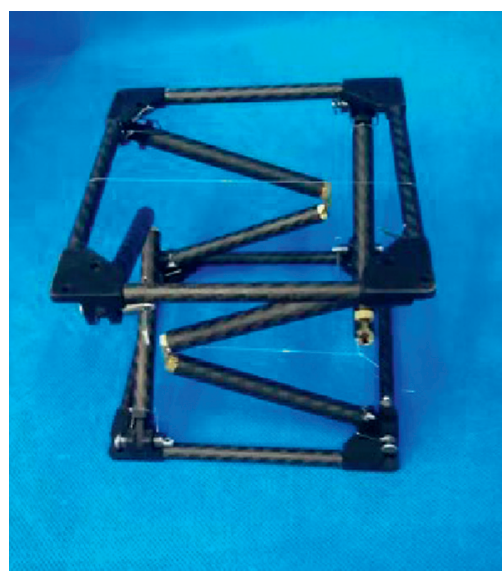

(b)

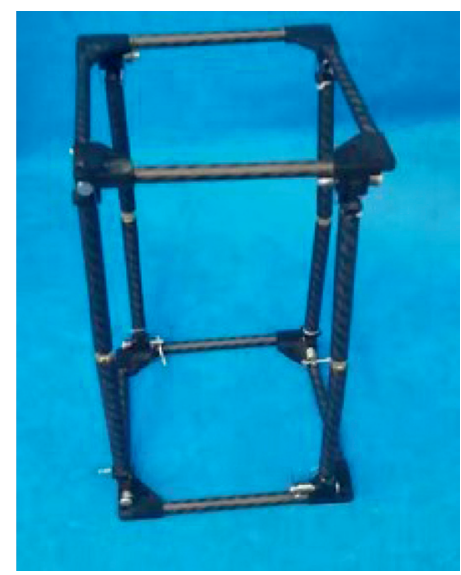

(c)

Figure 18: Deployment unit prototype.

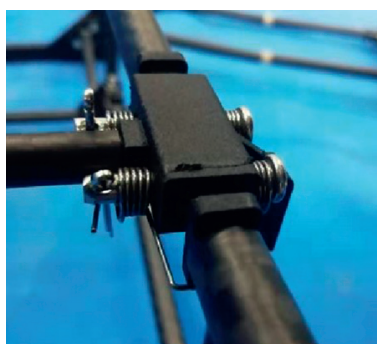

(a)

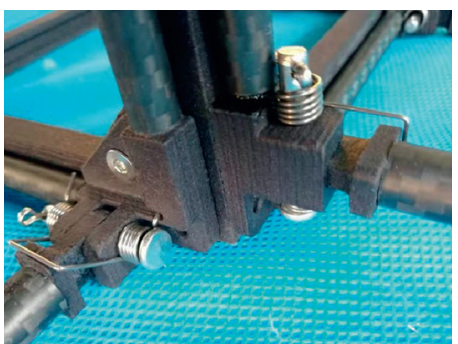

(b)

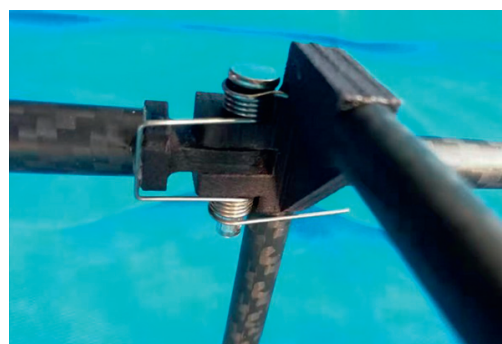

(c)

Figure 19: Deployment unit driver source installation location.

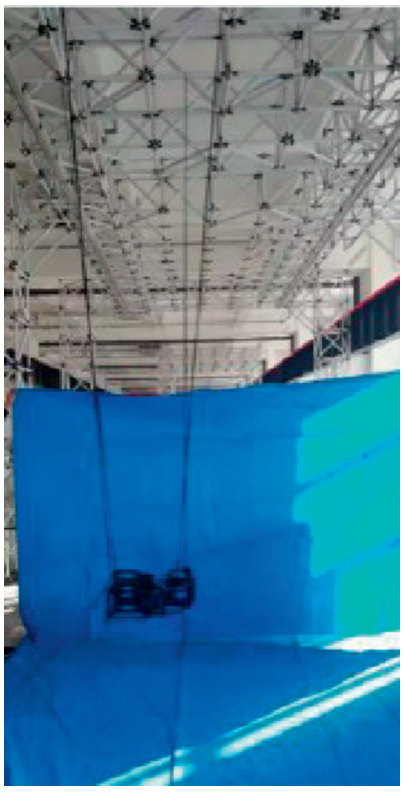

Figure 20: Microgravity test device.

rails, and the stretching mechanism is fixed to the two blocks with a cable. The block follows the movement during the unfolding process of the stretching mechanism, and the stretching mechanism is always maintained by the cable.
Figure 24 shows the organization's deployment process. The mechanism can be deployed under the action of only the turbofan drive, which verifies the feasibility of the deployable cabin system drive scheme in this study. 


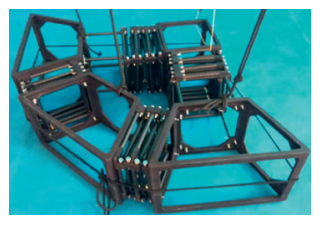

(a)

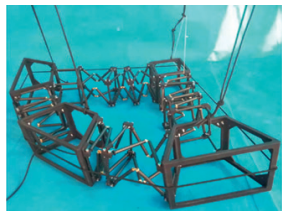

(b)

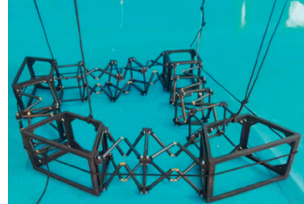

(c)

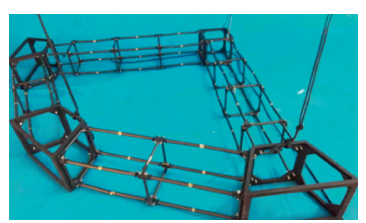

(d)

Figure 21: Deployment process of 19 1/4 ring prototypes.

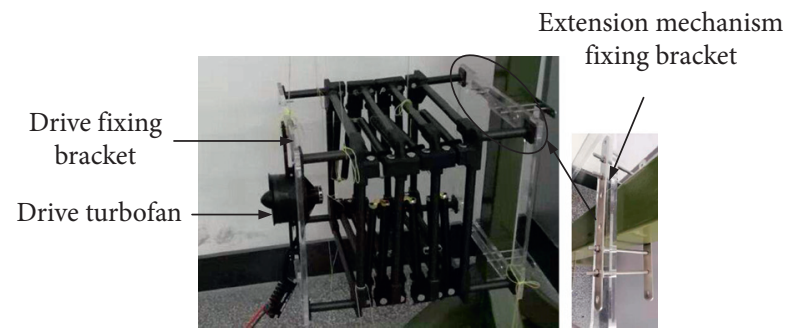

Figure 22: Prototype of longitudinal stretching mechanism.

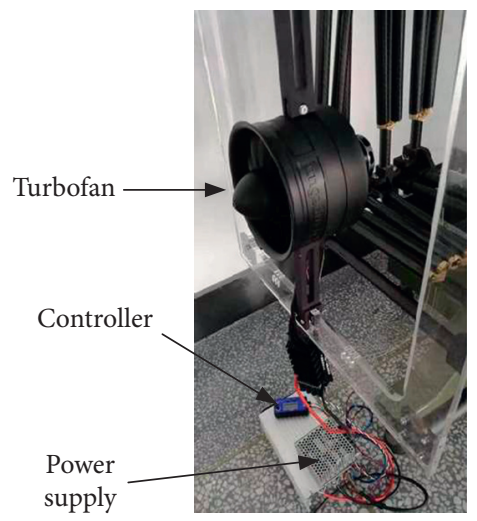

(a)

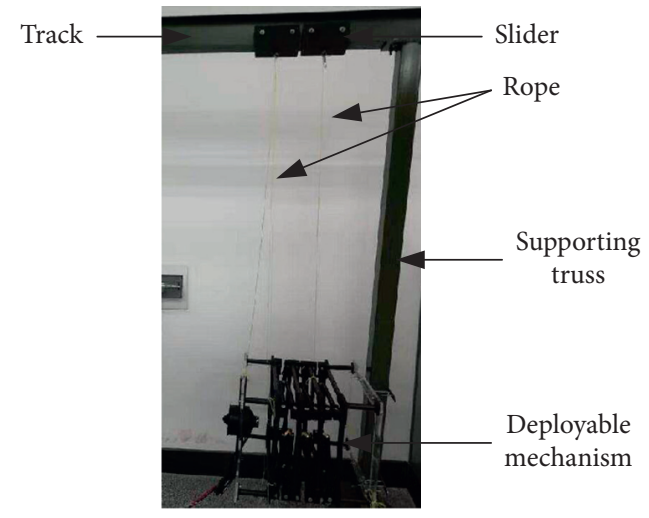

(b)

FIgURE 23: Stretching mechanism deployment experiment.

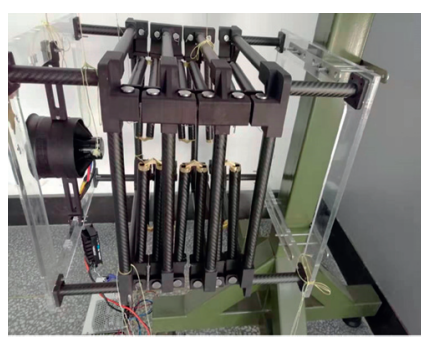

(a)

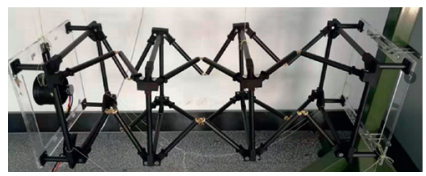

(c)

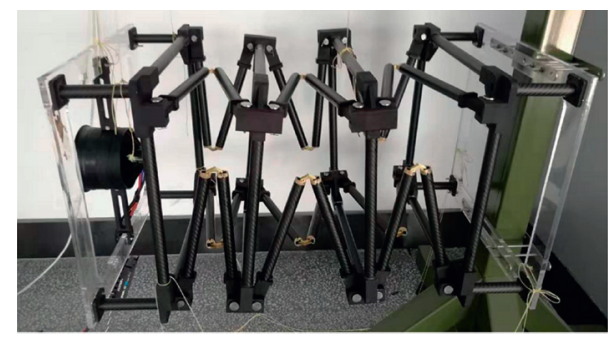

(b)

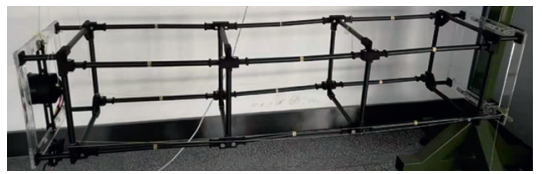

(d)

FIGURE 24: Stretching mechanism deployment process. 


\section{Conclusion}

This study designs an expandable space-expandable cabin system with a large stretch ratio, lightweight, and high rigidity. The main research content includes the selection of the basic unfolding unit, the construction of the cabin system, the structural design of the main components, such as the connecting hinge of the unfolding unit, the establishment of the mechanical equivalent model, and the development and testing of the principle prototype. The main conclusions include the following aspects:

(1) In this study, the basic deployment unit configuration is selected, and on this basis, the deployable cabin system scheme is established. The octagonal prism scheme is determined for the deployable cabin system. A new drive scheme of drag-and-drop cabin section system is proposed, and the unit connection hinge and unit structure are designed in detail.

(2) Based on the principle of energy equivalence, an equivalent continuous beam model of the expandable element is established. On this basis, the equivalent beam elements were assembled according to the topological relationship of the original model, and the equivalent mechanical model of the cabin system was obtained. Based on the established equivalent mechanical model of the system, the mechanical parameters, such as the axial tensile, bending, and torsional stiffness of the deployable cabin system, are derived. Through finite element simulation analysis, the axial tensile, bending, and torsional stiffness of the expandable cabin system are calculated. Comparing the obtained results with the theoretical analysis results, the errors are $10.06 \%$, $11.89 \%$, and $4.52 \%$. The error results verify the feasibility of the system equivalent model to analyze the statics characteristics of large-scale space structures. Similarly, the method of comparing theoretical calculation and finite element simulation is used to verify the feasibility of the system equivalent model to analyze the dynamic characteristics of large space structures.

(3) This study builds a quarter-ring scaled prototype to verify the rationality of the end structure of the cabin system. The prototype of the longitudinal extension mechanism consists of three units. Use the turbofan as a drive for experiments. Through experiments, the feasibility of the driving method and the deployment scheme proposed in this study are verified.

\section{Data Availability}

No data were used to support this study.

\section{Conflicts of Interest}

The authors declare that they have no conflicts of interest.

\section{Acknowledgments}

This project was supported by the Open Project of Space Structure and Mechanism Technology Laboratory of China Aerospace Science and Technology Group Co. Ltd., the SelfPlanned Task (no. SKLRS202004C) of State Key Laboratory of Robotics and System (HIT), the National Natural Science Foundation of China (52005123 and 51835002), the Joint Funds of the National Natural Science Foundation of China (grant no. U1637207), and the College Discipline Innovation Wisdom Plan in China (grant no. B07018). These supports are gratefully acknowledged by the authors.

\section{References}

[1] J. Chen, H. Nie, C. Chen, and W. Chen, "Design of Manned Lunar Module and research on some key technologies," Journal of Astronautics, vol. 35, no. 02, pp. 125-136, 2014.

[2] Z. Shen, "Application of inflatable expansion structure in spacecraft," Spacecraft Environment Engineering, vol. 04, pp. 323-329, 2008.

[3] D. Yu, "The development of China's lunar exploration project and its demand for space environment and materials research," Spacecraft Environment Engineering, vol. 27, no. 6, pp. 677-681, 2010.

[4] J. Duan, Y. Liu, Li Yan et al., "Preliminary study on orbit determination of change -4 relay satellite mission," Journal of Deep Space Exploration, vol. 5, no. 6, pp. 531-538, 2018.

[5] T. Sinn and O. Doule, "Inflatable structures for Mars base 10," in Proceedings of the International Conference on Environmental Systems, AIAA, San Diego, CA, USA, July 2012.

[6] Z. Szigetvari, J. Witt, J. Persson et al., Columbus Environmental Control System Tests: Verification of ATCS and ECLSS Performance, SAE, Warrendale, PA, USA, 2005.

[7] Z. Szigetvari and P. Vaccaneo, Columbus Integrated System Level ECS Test: Preparation, Conduction and Summary, SAE, Warrendale, PA, USA, 2003.

[8] M. M. Cohen, "First Mars habitat architecture," in Proceedings of the AIAA 2015 Space and Astronautics Forum, pp. 97-104, Pasadena, CA, USA, September 2015.

[9] M. M. Cohen, Mobile Lunar Base Concepts, pp. 845-853, American Institute of Physics, College Park, MD, USA, 2004.

[10] B. Imhof, W. Hoheneder, S. Ransom et al., "Building the test bed SHEE-a self deployable habitat for extreme environments lessons learnt and exploitation opportunities for the scientific community," in Proceedings of the 2013. NASA Human Integration Design Handbook, Newyork, NY, USA, November 2013.

[11] O. Doule and B. . Imhof, "Self-deployable habitat for extreme environments-universal platform for analog research," in Proceedings of the AIAA Space Forum 2014, pp. 4-7, San Diego, CA, USA, August 2014.

[12] D. L. Akin, "A parametric comparison of microgravity and macrogravity habitat," in Proceedings of the Design Elements, 42nd International Conference on Environmental Systems, pp. 15-19, San Diego, CA, USA, July 2012.

[13] M. R. Kathleen and B. E. Sheerri, Civilian American and European Surface Anthropometry Resource, SAE, Warrendale, PA, USA, 2016.

[14] Y. Wang, "The world's first inflatable activity capsule was successfully launched and its impact analysis," China Aerospace, vol. 7, pp. 16-22, 2006. 
[15] H. Guo, M. Li, X. Wang, Y. Zhang, and Na Lin, "Research and Application of MES in aerospace composite cabin assembly," Space Manufacturing Technology, vol. 03, pp. 46-49, 2018.

[16] W. Wang, F. Qiang, W. Shi, J. Chang, Z. Cheng, and Y. Liang, "Development of flexible inflatable capsule structure technology for spacecraft," Spacecraft Engineering, vol. 23, no. 01, pp. 103-109, 2014.

[17] B. D. Benjamin, N. C. Glenn, and B. S. David, "Lunar lander concepts for human exploration," Journal of Spaceand Rockets, vol. 45, no. 2, pp. 383-393, 2006.

[18] E. Seedhouse, "Bigelow expandable activity module," in Bigelow Aerospace Springer Praxis Books, pp. 87-98, Springer, Cham, Switzerland, 2014.

[19] N. Wells and E. Madaras, "The potential for health monitoring in expandable space modules. The bigelow expandable activity module on the ISS," in Proceedings of the 11th International Workshop on Structural Health Monitoring, Qingdao, China, September 2017.

[20] L. Guo, Z. Li, and Yi Qi, "Overall scheme and construction planning assumption of a comprehensive manned lunar base," Space Return and Remote Sensing, vol. 35, no. 6, pp. 1-10, 2014.

[21] Y. Chen, J. Yan, J. Feng et al., "PSO-based metaheuristic design generation of non-trivial flat-foldable origami tessellations with degree- 4 vertices," Journal of Mechanical DesignTransactions of the ASME, vol. 143, no. 1, Article ID 011703, 2021.

[22] Y. Chen, J. Yan, P. Sareh et al., "Feasible prestress modes for cable-strut structures with multiple self-stress states using particle swarm optimization," Journal of Computing in Civil Engineering, vol. 34, no. 3, Article ID 04020003, 2020.

[23] Y. Chen, Ke Liang, and X. Zhang, "Strategy for sensor number determination and placement optimization with incomplete information based on interval possibility model and clustering avoidance distribution index," Computer Methods in Applied Mechanics and Engineering, vol. 366, Article ID 113042, 2020.

[24] Y. Chen, "An adaptive sensor placement algorithm for structural health monitoring based on multi-objective iterative optimization using weight factor updating," Mechanical Systems and Signal Processing, vol. 151, Article ID 107363, 2021.

[25] Y. Chen, Ke Liang, and X. Zhang, "Sensor placement algorithm for structural health monitoring with redundancy elimination model based on sub-clustering strategy," $\mathrm{Me}$ chanical Systems and Signal Processing, vol. 124, pp. 369-387, 2019. 